\title{
A Coulomb collision algorithm for weighted particle simulations
}

\author{
Ronald H. Miller and Michael R. Combi \\ Space Physics Research Laboratory, Department of Atmospheric, Oceanic and Space Sciences, The University of \\ Michigan, Ann Arbor, Michigan
}

\begin{abstract}
A binary Coulomb collision algorithm is developed for weighted particle simulations employing Monte Carlo techniques. Charged particles within a given spatial grid cell are pair-wise scattered, explicitly conserving momentum and implicitly conserving energy. A similar algorithm developed by Takizuka and $A b e$ [1977] conserves momentum and energy provided the particles are unweighted (each particle representing equal fractions of the total particle density). If applied as is to simulations incorporating weighted particles, the plasma temperatures equilibrate to an incorrect temperature, as compared to theory. Using the appropriate pairing statistics, a Coulomb collision algorithm is developed for weighted particles. The algorithm conserves energy and momentum and produces the appropriate relaxation time scales as compared to theoretical predictions. Such an algorithm is necessary for future work studying self-consistent multi-species kinetic transport.
\end{abstract}

\section{Introduction}

The plasma medium, such as the ionosphere or plasmasphere, consists of electrons, ions, neutral atoms and molecules whose interaction is complex and at times difficult to understand completely. Particle simulations, however, can offer detailed information describing some of the interactions between the plasma constituents. Recently, particle simulations have been used to investigate polar outflow from the ionosphere [Wilson, 1992b; Miller et al., 1993b], and plasmaspheric refilling [Wilson et al., 1992a; Miller et al., 1993a]. These simulations are hybrid in nature, kinetic ions and fluid electrons, and incorporate Coulomb collisions through a binary Coulomb collision algorithm developed by Takizuka and Abe [1977, hereafter referred to as TA77]. Miller et al. [1993a,b] and Wilson et al. [1992a] modeled plasmaspheric refilling where Coulomb collisions alter the ballistic trajectories of ions, trapping $\mathrm{H}^{+}$in the plasmasphere, and thereby initiating the refilling of the plasmasphere. In order to simulate the actual flux from the ionosphere, for plasmaspheric refilling, each $\mathrm{H}^{+}$simulation particle corresponds to thousands of real particles. These hybrid simulations use unweighted particles where the simulation particles share equally the total particle density. Under these circumstances, the collision model of TA77 yields momentum and energy relaxation rates which agree quite favorably with theoretical predictions for early times, conserving momentum explicitly and energy implicitly.

In many plasma environments, however, simulation particles must be weighted in order to have good particle statistics while

\section{Copyright 1994 by the American Geophysical Union.}

Paper number 94GL01835

0094-8534/94/94GL-01835\$03.00 also not exceeding hardware limitations. A typical example can be found in the ionosphere where, depending on ionospheric condition, the $\mathrm{O}^{+}$density can be more than two orders of magnitude greater than the $\mathrm{H}^{+}$density at $800 \mathrm{~km}$. For these conditions, weighted particle simulations would best model the plasma environment. Wilson [1992b] modeled the topside collisional-to-collisionless transition region but considered $\mathrm{H}^{+}$as the only dynamic test particle species, while the $\mathrm{O}^{+}$species was considered a fixed field particle in diffusive equilibrium. The $\mathrm{O}^{+}$ density was an order of magnitude greater than the $\mathrm{H}^{+}$density; however, because $\mathrm{O}^{+}$was not a dynamic species, the simulation particles were not weighted. If Coulomb collisions are an important physical process which must be included, a new collision algorithm must be developed which is valid for weighted particles, enabling in theory a self-consistent solution for both $\mathrm{H}^{\mathbf{}}$ and $\mathrm{O}^{+}$.

In this paper, we present a new collisional model including the effect of small angle Coulomb collisions between different weighted populations, which will be used in future studies of selfconsistent multi-species kinetic transport. By careful consideration of pairing statistics, an algorithm can be developed so as to have the proper relaxation rates as compared to the unweighted simulation (TA77), along with conserving momentum and energy. Several plasma simulations are performed, varying the particle weightings and showing good agreement with theory.

\section{Theory}

\subsection{Takizuka and Abe Collision Model}

A binary collision algorithm was developed for Coulomb collisions, employing Monte Carlo techniques, in 1-D3v by TA77. The charged particles, in each spatial grid cell, are scattered off of each other, pair-wise, in the rest frame of the target particle, explicitly conserving momentum and implicitly conserving energy. The process is cumputationally intensive, but accurately models the exchange of energy and momentum between the colliding species. The procedure is to randomly pair particles in the same grid cell and to compute the deflection of their relative velocity, corresponding to that which would result from the cumulative effect of many small angle collisions experienced in a time $\Delta t$. The determination of the pairs is performed in two steps as discussed in TA77. Firstly, self-collisions are performed requiring each species in a given cell to be paired with itself. After self-collisions are taken into account, unlike collisions are performed by pairing unlike particles in each cell. For each pairing, the variable $\delta=\tan (\Theta / 2)$ (where $\Theta$ is the angular deflection of $u$ ) is chosen randomly from a Gaussian distributed with zero mean. The variance used in determining $\delta$ is given by $\left\langle\delta^{2}\right\rangle=\mathrm{e}_{\alpha}^{2} \mathrm{e}_{\beta}^{2} \mathrm{n}_{\mathrm{L}} \lambda \Delta \mathrm{t} /\left(8 \pi \varepsilon_{0}^{2} \mathrm{~m}_{\alpha \beta}^{2} \mathrm{u}^{3}\right)$ where $\alpha$ and $\beta$ refer to different species, $\varepsilon_{0}$ is the permitivity of vacuum, $n_{L}$ is the lower density between $n_{a}$ and $n_{b}, u$ is the relative velocity between 
species $\alpha$ and $\beta, m_{\alpha \beta}$ is the reduced mass, and $\lambda$ denotes the Coulomb logarithm. The change in velocity due to a binary collision can be determined given $\delta$ (see equation 4, TA77), while the total velocities are updated using $v_{\alpha, j}^{t+\Delta t}=v_{\alpha, j}^{t}+\left(m_{\alpha \beta} / m_{\alpha}\right) \Delta v_{\alpha, j}$ and $v_{\beta, j}^{t+\Delta t}=v_{\beta, j}^{t}-\left(m_{\alpha \beta} / m_{\beta}\right) \Delta v_{\beta, j}$ for species $\alpha$ and $\beta$, with $j$ corresponding to the spatial direction. TA77 performed several plasma simulations examining momentum and temperature relaxation and found good agreement between theory and numerical simulation.

\subsection{Binary Coulomb Collision Algorithm for Weighted Particle Simulations}

Consider a plasma where the two species have equal masses, $\mathrm{m}_{\alpha}=\mathrm{m}_{\beta}=\mathrm{m}$, the same fraction of the total density, $\mathrm{n}_{\alpha}=\mathrm{n}_{\beta}=\mathrm{n} / 2$, but species $\alpha$ has twice as many simulation particles as species $\beta$, $\mathbf{N}_{\alpha}=\mathbf{2 N}_{\beta}$. For this case, 1 simulation particle of species $\beta$ represents twice as much of the density as 1 simulation particle of species $\alpha$. When colliding these particles, using the binary algorithm of TA77, momentum is transferred between both species equally, even though one simulation particle is in a sense 2 times "larger" than the other, violating conservation of momentum and, subsequently, conservation of energy. The simplest way to correct this problem is to collide the particles, "kicking" the lighter particle all the time while "kicking" the heavier particle a fraction of the time, corresponding to the inverse weight of the heavier particle. This "rejection" method introduces a probability for colliding the heavier particles and is a common trick employed in Monte Carlo simulations. It assures that the microphysics is accurately represented, while macroscopic energy and momentum are conserved on the average By comparing a random number ( $r$ ) to the inverse weight, a binary probability is calculated which determines whether momentum is transferred to the heavier species, i.e., $P=1$ for $r<1 / w$ and $P=0$ for $r>1 / w$. Since we normalize the weights to the lightest species, $P=1$ for the lighter species for all times. Given this scenario, the updated particle velocities are modified to include the probability factor and can be written as $v_{\alpha, j}^{t+\Delta t}=v_{\alpha, j}^{t}+P_{\alpha}\left(m_{\alpha \beta} / m_{\alpha}\right) \Delta v_{\alpha, j}$ and $v_{\beta, j}^{t+\Delta t}=v_{\beta, j}^{t}-P_{\beta}\left(m_{\alpha \beta} / m_{\beta}\right) \Delta v_{\beta, j}$. For the case discussed above, where species $\beta$ is twice as large as species $\alpha, P_{\alpha}=1$ for all times while $P_{\beta}=1$ or $P_{\beta}=0$ provided the random number satisfies $r<$ 0.5 or $r>0.5$, respectively. The methodology conserves energy and momentum on an average and has been used successfully in modeling cloud-cloud collisions in stellar dynamics (Roberts and Stewart [1987] and references therein). Without this probability factor, one would transfer too much momentum to the heavier particles (since they are weighted) and, subsequently, momentum and energy would not be conserved.

A weighting factor must also appear in the expression for the variance of $\delta$ which comes from under-colliding or over-colliding the plasma particles in the weighted system. The introduction of this weight can be seen by comparing the pairing statistics between an unweighted and weighted particle simulation. Table 1 contains the corresponding collision pairs for an unweighted plasma of $N_{\alpha}=N_{\beta}=4000$ particles and a weighted plasma of $N_{\alpha}=3 N_{\beta}=6000$ particles (note: each species still represents half of the total density). In the weighted simulation, the number of $\alpha \alpha$ collision has increased with a decrease in $\beta \beta$ and $\alpha \beta$ collisions. Hence, the magnitude of $\left\langle\delta^{2}>\right.$ must decrease for $\alpha \alpha$ collision and increase for $\alpha \beta$ and $\beta \beta$ collision in order to have the same cumulative scattering, along with a similar relaxation time scale, as in the unweighted particle simulation.

Generalizing the problem for an arbitrary number of simulation
Table 1: Comparison of possible collision pairs for unweighted and weight particle simulations.

\begin{tabular}{ccc} 
Collision Pairs & $\begin{array}{c}\text { Unweighted Particles } \\
N_{\alpha} / N_{\beta}=4000 / 4000=1\end{array}$ & $\begin{array}{c}\text { Weighted Particles } \\
N_{\alpha} / N_{\beta}=6000 / 2000=3\end{array}$ \\
\hline$N_{\alpha \alpha}$ & 1000 & 1500 \\
$N_{\beta \beta}$ & 1000 & 500 \\
$N_{\beta \alpha}$ & 1000 & 1500 \\
$N_{\alpha \beta}$ & 1000 & 500 \\
\hline
\end{tabular}

particles per plasma population, and for when each species may represent a different fraction of the total particle density, the total number of collision pairs in the unweighted simulation can be determined as

$$
\begin{aligned}
& \mathrm{N}_{\alpha \alpha}=\left(\frac{N}{2}\right) \frac{\chi^{2}}{(1+\chi)^{2}} \quad \mathrm{~N}_{\alpha \beta}=\left(\frac{N}{2}\right) \frac{\chi}{(1+\chi)^{2}} \\
& \mathrm{~N}_{\beta \alpha}=\left(\frac{N}{2}\right) \frac{\chi}{(1+\chi)^{2}} \quad \mathrm{~N}_{\beta \beta}=\left(\frac{N}{2}\right) \frac{1}{(1+\chi)^{2}}
\end{aligned}
$$

where, for the weighted simulation, the collision pairs become

$$
\begin{array}{ll}
N_{\alpha \alpha}^{w}=\frac{(N / 2) r_{s} \chi}{\left(1+r_{s}\right)(1+\chi)} & N_{\alpha \beta}^{w}=\frac{(N / 2) \chi}{\left(1+r_{s}\right)(1+\chi)} \\
N_{\beta \alpha}^{w}=\frac{(N / 2) r_{s}}{\left(1+r_{s}\right)(1+\chi)} & N_{\beta \beta}^{w}=\frac{(N / 2)}{\left(1+r_{s}\right)(1+\chi)}
\end{array}
$$

where $\mathbf{N}$ is the total number of particles, $\chi=$ density ratio $=n_{\alpha} / n_{\beta}$ and $\mathbf{r}_{\sigma}=$ simulation particle ratio $=N_{\alpha} N_{\beta}$. The above collision pairs were determined by conserving the total number of simulation particles and by pairing individual particles from an ensemble. By forming ratios of the weighted to unweighted collision pairs, i.e., $w_{\alpha \alpha}=N_{\alpha \alpha}^{w} / N_{\alpha \alpha}, w_{\alpha \beta}=N_{\alpha \beta}^{w} / N_{\alpha \beta}, w_{\beta \alpha}=N_{\beta \alpha}^{w} / N_{\beta \alpha}, w_{\beta \beta}=N_{\beta \beta}^{w} / N_{\beta \beta}$, collision weights are produced measuring the change in the number of collisions for the weighted simulation. Using the parameters given in Table 1 , the weights become $w_{\alpha \alpha}=3 / 2$, $w_{\alpha \beta}=1 / 2, \quad w_{\beta \alpha}=3 / 2$ and $w_{\beta \beta}=1 / 2$ with $\chi=1$. These weights imply that in the weighted simulation (1) $3 / 2$ too many $\alpha \alpha$ collisions occur, (2) $\alpha \beta$ collisions are $1 / 2$ as frequent, (3) $\beta \alpha$ collisions are $3 / 2$ as frequent, and (4) $1 / 2$ as many $\beta \beta$ collisions occur as in the unweighted simulation.

Two modifications must be performed on the expression for the variance (1) in order to correctly describe Coulomb collision in a weighted plasma simulation. The first modification is due to the change in the number of scattering events for a weighted simulation (see Table 1). If the number of scattering events increases by " $w$ ", then $\left\langle\delta^{2}\right\rangle$ must decrease by this factor producing the appropriate relaxation time scales (note: the magnitude of $\left\langle\delta^{2}\right\rangle$ is proportional to the rate at which the plasma equilibrates). The variance is replaced by three new expressions for the variance depending on the pairing of the species, i.e., $\left.<\delta^{2}\right\rangle_{\alpha \alpha}=\left\langle\delta^{2}\right\rangle / w_{\alpha \alpha}, \quad\left\langle\delta^{2}\right\rangle_{\alpha \beta}=\left\langle\delta^{2}\right\rangle_{\beta \alpha}=\left\langle\delta^{2}\right\rangle /\left(w_{\alpha b} w_{\beta \alpha}\right)$ and $<\delta^{2}>_{\beta \beta}=<\delta^{2}>/ w_{\beta \beta}$. Pairing statistics are responsible for the second modification of the variance where the lower density, $\mathbf{n}_{\mathbf{L}}$, is replaced by the total density. Unlike TA77, all particles in a given cell, independent of the species type, are paired only once and then scattered using the modified variances with the total 
density. This can introduce a substantial decrease in computational time as compared to TA77, while TA77 must sort due to species and cell location, then perform self-scattering (same species type) and unlike particle scattering (see TA77 for further details).

The probability factors in the momentum exchange equations $\left[P_{\alpha}\right.$ and $\left.P_{\beta}\right]$ are determined by normalizing to the smallest weighted species. If $w_{\alpha \alpha}>w_{\beta \beta}$, then $P_{\alpha}=1$ with $P_{\beta}=1$ for random numbers ( $r$ ) such that $r<w_{\beta \beta} / w_{\alpha \alpha \alpha}$, or $P_{\beta}=0$ for $r>w_{\beta \beta} / w_{\alpha \alpha}$. Similarly, for $w_{\beta \beta}>w_{\alpha \alpha}$ then $P_{\beta}=1$ with $P_{\alpha}=1$ for random numbers (r) such that $\mathrm{r}<\mathrm{w}_{\alpha \alpha} / w_{\beta \beta}$, or $\mathrm{P}_{\alpha}=0$ for $\mathrm{r}>\mathrm{w}_{\alpha \alpha} / w_{\beta \beta}$.

\section{Results}

In this section, the results primarily focus on the temperature relaxation of a multi-species plasma as presented in TA77. TA77 consider two species, electrons and ions, initially at two different temperatures and compared the theoretical relaxation time scales to numerical time scales using their Coulomb collision model. We focus on one range of parameters (cf Figure 9b of TA77), however, the results are general and applicable to other parameter regimes.

A single cell particle-in-cell simulation including Coulomb collisionsis used to investigate temperature equilibration for an electron-ion plasma. The particles are distributed uniformly within the cell and periodic boundary conditions are assumed. The simulation parameters for the density (n), temperature (T), and mass $(m)$ are the following, $n_{i}=n_{a}=n, T T_{i}=2 T_{0}$ and $m_{i}=4 m$. where the subscript " $\mathrm{i}$ " and "e" refer to the ion and electron species, respectively. The total density was assumed to be $n_{T}=2 n=1.0 \times 10^{6} \mathrm{~cm}^{-3}$ with an electron temperature of $T_{e}=200 \mathrm{eV}$. The time step used in the simulations is $\Delta t=0.001 v_{0}^{-1}$ for a total of 10000 time steps. All parameters remain constant in the simulations except for the number of simulation particles. The equilibration of temperatures can be expressed analytically with the following form

$$
\frac{d\left(T_{i}-T_{e}\right)}{d t}=-2 v_{e q}\left(T_{i}-T_{e}\right)
$$

where $v_{e q}=\left(8 / 3 \pi^{1 / 2}\right)\left(m_{e} / m_{i}\right)\left[1+m_{e} / m_{i} \cdot\left(T_{i} / T_{e}\right)\right]^{-3 / 2} v_{0}$ is the temperature equilibration frequency with the collision frequency $v_{0}$ satisfying $v_{0}=e^{4} n \lambda /\left(8 \sqrt{2} \pi \varepsilon_{0}^{2} m_{0}^{1 / 2} T_{e}^{3 / 2}\right)$. Equation (1) can be numerically integrated and compared to the relaxation rates obtained by using the collision algorithm.

Figure 1 shows the temperature relaxation between ions and electrons, initially at different temperatures, as a function of normalized time. The simulation uses 30000 particles with 15000 ions and 15000 electrons. Since the densities are the same, the electrons and ions have the same weighting which allows for a comparison between TA77 and the new weighted collision algorithm (hereafter referred to as MC94). Figure 1a shows a comparison between the numerical relaxation rates from TA77 (solid) and MC94 (dash-dot) and the theoretical predictions (dot) for early times, $t<0.4 v_{0}^{-1}$. The numerical and theoretical relaxation rates are approximately equal and are in good agreement with Figure $9 \mathrm{~b}$ of TA77. However, as displayed in Figure $1 \mathrm{a}$ and $1 \mathrm{~b}$, the theoretical and numerical relaxation rates diverge times, $t>0.2 v_{0}^{-1}$, where the numerical curves have a slower relaxation as compared to theoretical predictions. This dependence is not easily seen in Figure 9b of TA77 since TA77 only use a total of 6000 particles. Using 30000 particles, Figure 1
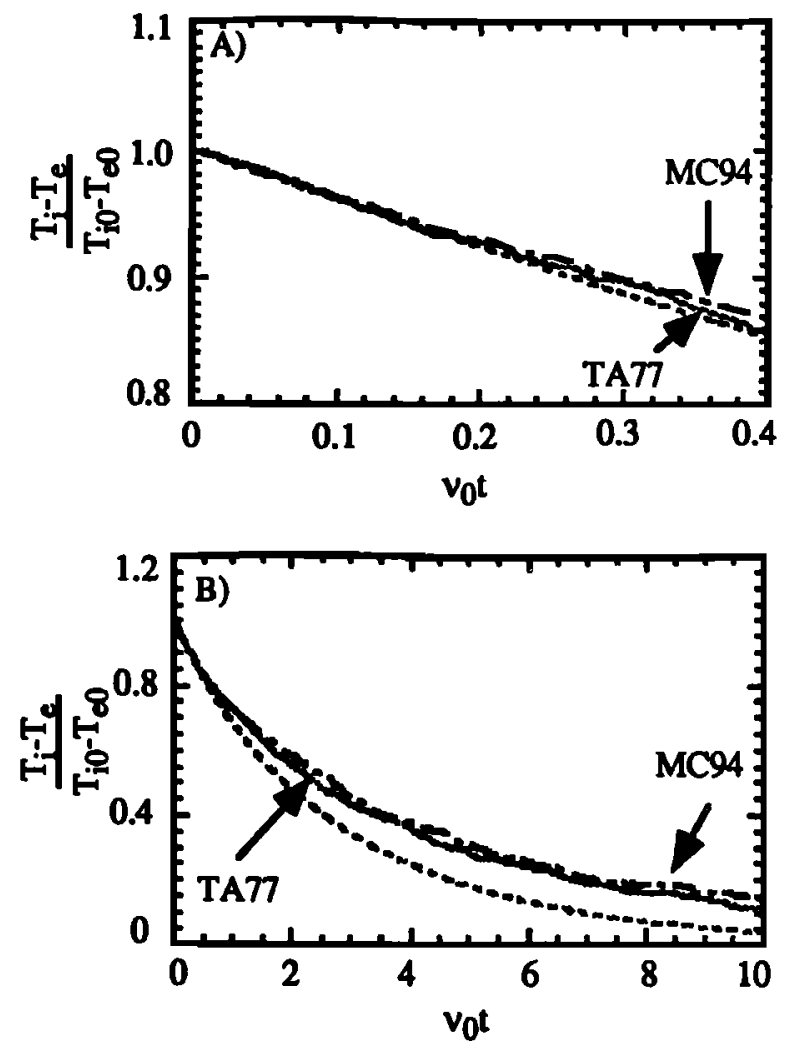

Figure 1. The equilibration of ion and electron temperatures for $m_{i} / m_{e}=4, T_{i} / T_{c}=2$. Figure 1 a shows the early time $\left(t<0.4 v_{0}^{-1}\right)$ temperature equilibration, while Figure $1 b$ corresponds to late time $\left(t>0.4 v_{0}^{-1}\right)$. The theoretical prediction, (1), is the dashed curve whereas the solid curve is the TA77 simulation.

show smooth divergence of TA77 and MC94 as early as $0.2 v_{0}^{-1}$ from the theoretical predictions. By $5.0 \mathrm{v}_{0}^{-1}$, the three curves have approximately the same slope showing that the numerical schemes (MC94 and TA77) equilibrate slower than (1) but will produce the same asymptotic temperature as (1).

As shown in Figure 1, the relaxation rates agree quite favorably for small times (Figure 1a) for non-equilibrium conditions; however, for times greater than $0.2 \mathrm{v}_{0}^{-1}$, the numerical and theoretical results diverge (Figure $1 \mathrm{a}$ and $1 \mathrm{~b}$ ). The reason for this discrepancy is due to the non-Maxwellian features which develop as the plasma starts to equilibrate. Equation (1) implicitly assumes Maxwellian particle distributions and that higher order moments can be neglected. Since the Coulomb cross section is highly velocity dependent, the particle distributions do not maintain a Maxwellian form during the energy exchange process. Hence, (1) does not suitable describe the equilibration process except for very early or very late times. This same behavior was found by TA77 but never discussed because they focused on early time scales dynamics (see Figure 8 of TA77). In the future, the numerical schemes should be compared to higher order moment calculations or Fokker-Planck solutions as a further test for the range of validity and application.

Concentrating on early time dynamics and using MC94, Figure 2 shows temperature equilibration rates for three different weightings as a function of normalized time. The theoretical prediction, (1), corresponds to the dashed curve while the numerical cases have the following weightings: (1) the solid curve has $r_{\sigma}=15000 / 15000=1$, (2) the dot-dash curve has $r_{\sigma}=22500 / 7500=3$, and (3) the dot-dot-dash curve has 


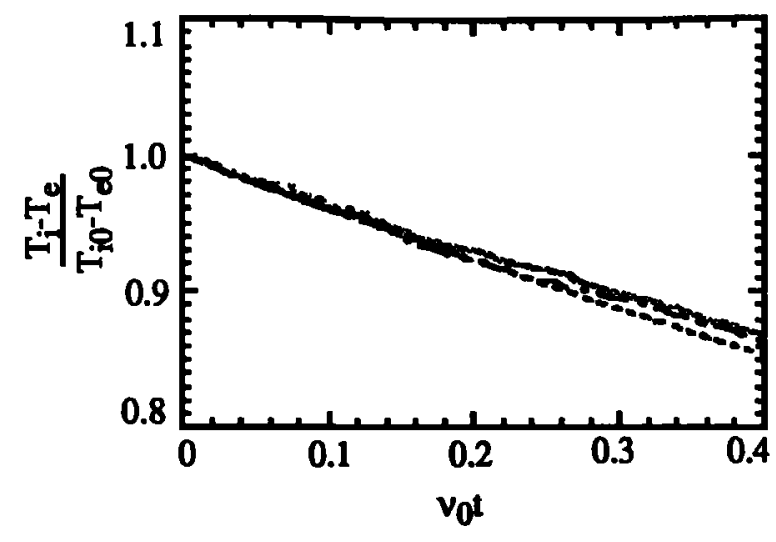

Figure 2. The equilibration of ion and electron temperatures for weighted plasma simulations given $m_{i} m_{c}=4$, and $T_{i} / T_{c}=2$. The theoretical prediction is dashed with the numerical equilibration given by (1) a solid curve for $r_{s}=15000 / 15000$, (2) a dot-dash curve for $r_{z}=22500 / 7500$, and (3) a dot-dot-dash curve for $r_{3}=25000 / 5000$.

$r_{\sigma}=25000 / 5000=5$ where the total number of simulation particles is 30000 with $\chi=1$. The results agree so favorably that the curves are almost indistinguishable. The algorithm conserves energy and momentum and has the appropriate relaxation rates as compared to theory for early times. The numerical routine has been tested for various densities $(\chi)$ and simulation particle ratios $\left(r_{8}\right)$ and found to agree with theory and TA77.

\section{Conclusion}

Many geophysical applications involve plasmas with densities which differ by orders of magnitude, such as in the modeling of ionospheric outflow and plasmaspheric refilling. Recently, kinetic simulations have been used to examine polar outflow and plasmaspheric refilling [Miller et al., 1993a; Miller et al., 1993b; Wilson et al., 1992a; Wilson, 1992b], incorporating the effects of Coulomb collisions using a method developed by TA77. The application of these kinetic simulations is limited to regions where the particle densities of each species are comparable. For particle densities which are not comparable, such as in the Earth's ionosphere, particle weighting is essential in order to efficiently simulate the plasma dynamics. Once weighted particles are used, the collisional algorithm of TA77 does not conserve energy and momentum. Thus, in order to perform a multi-species kinetic simulation of the Earth's ionosphere, or any geophysical region where collisional processes are important, a new Coulomb collision algorithm is needed which conserves energy and momentum.

We have developed a weighted collision algorithm (MC94) which conserves energy and momentum, for arbitrary particle densities. By considering pairing statistics between the weighted and unweighted simulations, probability weights are determined and a "rejection" method used which allows for the numerical modeling of Coulomb collisions for arbitrary particle weighting. This algorithm is valid for unweighted as well as weighted simulations and is significantly faster ( $\sim$ a factor of 2 or more) than TA77 for unweighted simulations. MC94 group all particles together only once and scatter the particles by pairing particles randomly regardless of the species type, thus MC94 include self collisions as well as unlike particle collisions at the same time. TA77, however, group and scatter each species (self-collisions) and then group and scatter unlike particles. MC94 was found to agree quite favorably with theoretical predictions (Figure 1 and 2) for early times, $t<0.4 v_{0}{ }^{-1}$, and with TA77 for all times provided the simulations are unweighted.

A discrepancy between the theoretical predictions and the numerical results, using both MC94 and TA77, were obtained for times greater than $0.4 \mathrm{v}_{0}^{-1}$ and can be initially observed as early as $0.2 v_{0}^{-1}$. The theoretical predictions (1) are predicated on the assumption that higher order moments (heatflow etc...) are negligible and that the particle distributions remain near a Maxwellian as the plasma equilibrates. The Coulomb cross section is highly velocity dependent and, therefore, as the plasma equilibrates, non-Maxwellian features develop and the applicability of (1) is speculative. More research is needed in comparing MC94 and TA77 to either higher order fluid equations (energy equation including heatflow etc.) or to a Fokker-Planck solution to test the range of applicability for these binary numerical scattering algorithms.

Acknowledgments. The authors wish to thank D. Winske, C. E Rasmussen, R. Roussel-Dupré and T. Gombosi for useful discussions. This work was supported at the University of Michigan by the National Aeronautics and Space Administration (NASA) grant NAGW-1619 and NAGW-3417 and by the National Science Foundation under contract ATM-9111440.

\section{References}

Miller, R. H., C. E Rasmussen, T. I. Gombosi, and D. Winske, Hybrid simulations of plasmaspheric refilling including convection and injection, Adv. Space Res., 13, (4)117-(4)120, 1993a.

Miller, R. H., C. E Rasmussen, T. I. Gombosi, G. Khazanov, and D. Winske. A kinetic simulation of plasma flows in the inner magnetosphere, J. Geophys. Res., 98, 19301-19313 1993b.

Roberts, W. W., Jr., and G. R. Stewart, The role of orbital dynamics and cloud-cloud collisions in the formation of the giant molecular clouds in global spiral structures, Astrophys. J., 314, 10-32, 1987.

Spitzer, L., Jr., Physics of Fully Ionized Gases, Chap. 5, Interscience, New York, 1956.

Takizuka, T. and H. Abe, A binary collision model for plasma simulation with a particle code, J. Comp. Phys., 25, 205-219, 1977.

Wilson, G., J. L. Horwitz, and J. Lin, A semikinetic model for early stage plasmasphere refilling 1. Effects of Coulomb collisions, J. Geophys. Res., 97, 1109-1119, 1992a.

Wilson, G., Semikinetic modeling of the outflow of ionospheric plasma through the topside collisional to collisionless transition region, $J$. Geophys. Res., 97, 10551-10565, $1992 \mathrm{~b}$.

R. H. Miller and M. R. Combi, Space Physics Research Laboratory, Department of Atmospheric, Oceanic and Space Sciences, The University of Michigan, Ann Arbor, MI 48109-2143. (e-mail: miller@spric.sprl.umich.edu.; combi@sprlc.spr.umich.edu.

Received: November 29, 1993; revised: May 3, 1994; accepted: June 26, 1994 